\section{Opdivo's lung cancer aspirations dashed}

Bristol-Myers Squibb's programmed cell death protein-1 (PD-1)-targeting immunotherapy Opdivo (nivolumab) failed to benefit patients with non-small cell lung cancer (NSCLC) in a first-line setting compared with chemotherapy. The treatment failed regardless of PD-L1 (PD-1 ligand) protein expression used as a exploratory predictive biomarker measured on patients' tumor cells. The company presented the phase 3 CHECKMATE-026 trial data in October at the European Society for Molecular Oncology (ESMO) annual meeting in Copenhagen. At the same meeting, favorable results in NSCLC released by competitors prompted a $10 \%$, or \$9.4-billion; drop in the New York-based firm's stock valuation. Opdivo had previously received approval from the US Food and Drug Administration as a second-line therapy in NSCLC, that is, for use in patients with metastatic disease that has progressed during or following initial treatment. The approval is for all patients regardless of PD-L1 (PD-1 ligand) expression-unlike rival Merck's Keytruda (pembrolizumab), for example, also approved as a second-line lung cancer treatment. BristolMyers Squibb disclosed in August that Opdivo had not improved progression-free survival in CHECKMATE-026 when compared with chemotherapy in patients with more than $5 \%$ PD-L1 protein expression on tumor cells. An analysis presented at ESMO showed the drug did not even benefit patients whose tumors expressed more than 50\% PD-L1 and, in some groups, the patients did less well on Opdivo than with chemotherapy. At the same time, Kenilworth, New Jersey-based Merck showed 10.3 months median progression-free survival with Keytruda in first-line NSCLC compared with six months on chemotherapy. The US Food and Drug Administration (FDA) is expected to approve before year-end.

Another challenger to Opdivo's aspirations in the lung cancer market is anti PD-L1 antibody Tecentriq (atezolizumab). The drug, owned by S. San Francisco-based Genentech, received FDA approval October 18 to treat patients with metastatic NSCLC, regardless of PD-L1 expression, whose disease has progressed during or following platinum-containing chemotherapy or in some cases after a targeted therapy. Phase 3 data presented at the conference in October showed median progression-free survival of 13.8 months for Tecentriq versus 9.6 months for Taxotere/Docefrez (docetaxel) chemotherapy, with better overall results in trial participants with high PD-L1 expression on tumor cells or tumor-infiltrating cells.

\section{Hope for anti-amyloid antibodies surges, yet again}

In late August, researchers at Cambridge, Massachusetts-based Biogen published full results from the phase $1 \mathrm{~b}$ clinical trial of aducanumab showing a striking dose-dependent reduction in brain amyloid- $\beta$ (A $\beta)$ aggregates, in patients with the earliest stages of Alzheimer's disease (Nature 537, 50-56, 2016). When these phase 1 data became available in 2015, not only did they convince Biogen to jump into phase 3 with aducanumab, a human monoclonal antibody that selectively targets A $\beta$ (Nat. Biotechnol. 33, 438, 2015), but they also sparked renewed confidence and enthusiasm in the entire field. Most notably, S. San Francisco, California-based Genentech (owned by Roche), pushed forward its own anti-A $\beta$ antibody crenezumab into phase 3 despite the drug's failure to reach its primary efficacy endpoints in phase 2. Biogen's clinical data have also boosted hope for positive results from a phase 3 trial of Indianapolis-based Eli Lilly's solanezumab in mild Alzheimer's disease, which are expected before the end of 2016 and represent the next inflection point in Alzheimer's disease progress and enthusiasm.

Together, these data suggest screening patients with improved imaging and genomic diagnostic tools and intervening in the earlier stages of the disease (Box 1) could yield successes in clinical trials with anti-amyloid agents where previous attempts have failed.

The rewards for an approved drug in this indication could be substantial. Datamonitor Healthcare analyst Maha Elsayed forecasts that $\mathrm{A} \beta$ antibodies from Lilly, Roche and Biogen could reach combined sales of more than $\$ 9$ billion by 2024 . What's more, if ongoing phase 3 trials show a slowing of cognitive for the idea of amyloid as a central actor in Alzheimer's disease. The deposition of $A \beta$ plaques and neurofibrillary tangles in the brain, accompanied by synaptic dysfunction and neurodegeneration, is the hallmark of Alzheimer's disease. The amyloid hypothesis posits that clearing such aggregates should mitigate or reverse the disease. But so far, antibody-based immunotherapy against $A \beta$ to trigger its clearance has been unsuccessful.

Despite repeated setbacks, $A \beta$ antibodies have attracted large investments for nearly a decade. In 2009, Johnson \& Johnson paid Elan $\$ 1.5$ billion for the now defunct antibody bapineuzumab (Nat. Biotechnol. 27, 679-681, 2009). Both bapineuzumab as well as Lilly's solanezumab failed in large and expensive phase 3 studies in mild-to-moderate Alzheimer's disease. But evidence that anti-A $\beta$ antibodies might work better in earlier stages of the disease has encouraged Lilly and others to move upstream, even as far as testing their therapies prior to $A \beta$ plaque formation. "There's an increasing realization that amyloid plaques begin to form at least 15 years before the development of Alzheimer's symptoms," says Eric Siemers, distinguished medical fellow for Lilly's Alzheimer's team. The phase 3 trial data for solanezumab is expected before the end of 2016.

Genentech and Lausanne, Switzerlandbased AC Immune launched in 2013 a clinical trial with anti-A $\beta$ crenezumab in individuals decline, they would offer compelling support

\section{Box 1 Beyond $A \beta$}

Therapeutics that prevent amyloid from forming in the first place by blocking the $\beta$-secretase cleaving enzyme (BACE) are also gaining momentum. The small-molecule BACE inhibitors interfere with one of the enzymes that chops up the amyloid precursor protein, thereby preventing creation of A $\beta$ peptide (Nat. Biotechnol. 33, 115-116, 2015). Results of a pivotal trial of Merck's MK-8931 in mild-to-moderate patients, the most advanced BACE inhibitor in development, are expected out in mid-2017. Although Merck didn't screen patients with PET imaging or determine amyloid in cerebrospinal fluid, a phase 2/3 trial of Lilly's and London-based AstraZeneca's BACE inhibitor AZD3293 that began in late 2014 did. Several biopharma companies including Lilly, Roche and Biogen are also developing antibodies against tau protein, which aggregates in the brains of Alzheimer's patients, appearing years after $A \beta$ plaques. All anti-tau antibodies are in early-stage clinical trials. The uptick in sentiment around $A \beta$ antibodies arrives even as a different class of potential Alzheimer's therapies has foundered. Idalopirdine, a 5-HT6 receptor antagonist from Copenhagen-based $\mathrm{H}$. Lundbeck and partner Otsuka in Tokyo, failed to hit its primary endpoint in a phase 3 trial in mild-to-moderate Alzheimer's, Lundbeck said in late September, and a similar molecule from New York-based Pfizer failed earlier in the year. 